\section{Finding cases of angle-closure glaucoma in clinic setting using a newly developed instrument}

\author{
Abstract \\ Purpose To validate the applicability of \\ a newly developed, noncontact scanning \\ peripheral anterior chamber depth analyzer \\ (SPAC) for screening eyes at the risk of angle- \\ closure glaucoma (ACG). \\ Subjects and methods All glaucoma patients \\ who visited the University of Yamanashi \\ Hospital from February through May 2003 \\ were enrolled, except those with aphakic eye \\ or pseudophakic eye. Of the 552 enrolled \\ patients, 48 with ACG or narrow angles \\ requiring laser iridotomy $(\mathrm{LI})$ were categorized \\ as patients with high-risk ACG eyes, and \\ those with open angle were categorized as \\ patients with control eyes. In all, 20 patients \\ with ACG or narrow angles requiring \\ prophylactic LI, who were followed up by an \\ independent private ophthalmic clinic, were \\ enrolled for threshold analysis. \\ Nonophthalmologists measured anterior \\ chamber depth and the averaged values \\ of three measurements were employed \\ for analysis. Threshold analysis and \\ discriminant analysis were employed for \\ determining the sensitivity and specificity \\ of SPAC for diagnosing eyes with \\ high-risk ACG. \\ Results SPAC distinguished well the high- \\ risk ACG eyes from the control eyes, and one \\ of the most useful criteria for screening is as \\ follows: any of the four measured points \\ should exceed $95 \%$ confidence interval, and \\ sensitivity and specificity should be 97.6 and \\ $83.5 \%$, respectively. \\ Conclusion SPAC is thought to be useful for \\ detecting eyes at the risk of ACG by \\ nonophthalmologists. \\ Eye (2006) 20, 319-324. doi:10.1038/sj.eye.6701869; \\ published online 8 April 2005
}

K Kashiwagi ${ }^{1}$, F Kashiwagi ${ }^{2}$, Y Hiejima ${ }^{3}$

and S Tsukahara'

Keywords: angle-closure glaucoma; screening; anterior chamber depth

\section{Introduction}

Angle-closure glaucoma (ACG) is one of the leading causes of acquired blindness, and there are a large number of patients with ACG, particularly in Asian countries. ${ }^{1-6}$ Although vision deteriorates within a short time after the onset of acute ACG in some cases, because there are neither subjective symptoms nor abnormalities, such as glaucomatous optic neuropathy or elevated intraocular pressure (IOP), until the actual onset, it is rarely detected in regular health examinations. On the other hand, as other forms of glaucoma, such as primary open angle glaucoma (POAG), occur and progress very slowly, abnormalities may be detected in fundus examinations and other ophthalmologic examinations. In contrast to POAG that does not have any form of preventive treatment before its onset and requires periodic follow-up and/or treatment to prevent further deterioration throughout the patient's life after its onset, the onset of ACG can be prevented in many cases by laser iridotomy (LI), and the effects of that treatment can potentially last throughout the patient's life. Despite the availability of preventive treatment, however, because it is difficult for nonophthalmologists to detect eyes with possible ACG, named primary angle closure (PAC) ${ }^{7}$ PAC is hardly detected in regular health examinations. As a result, many patients suffer from serious visual impairment because of acute ACG. With this in mind, we have developed a noncontact scanning peripheral anterior chamber depth analyzer (SPAC), which
${ }^{1}$ Department of Ophthalmology, University of Yamanashi, Faculty of Medicine, Tamaho Yamanashi, Japan

${ }^{2}$ Kashiwagi Ophthalmic Clinic, Kofu, Yamanashi, Japan

${ }^{3}$ Department of Data Science, University of Yamanashi Faculty of Medicine, Tamaho,

Yamanashi, Japan

Correspondence: K Kashiwagi, Department of Ophthalmology, University of Yamanashi Faculty of Medicine, 1110 Shimokato, Tamaho, Yamanashi 409-3898, Japan Tel: +81 55273 9657; Fax: +81552736757.

E-mail: kenjik@ yamanashi.ac.jp

Received: 19 October 2004 Accepted: 15 February 2005 Published online: 8 April 2005

Propriety interest: The instrument is applied for Japanese Patent (Application No. 2003111322) 
is a noninvasive technique that enables physicians and health care staff other than ophthalmologists to easily and accurately detect eyes with narrow angle. ${ }^{8}$ In this study, the usefulness of SPAC in ACG screening was assessed by investigating its sensitivity and specificity for detecting PAC eyes, focusing on patients already suffering from glaucoma and of whom the type of glaucoma had been correctly diagnosed.

\section{Subjects and methods}

This study was conducted in accordance with the Helsinki Declaration with approval from the Ethics Committee of the University of Yamanashi, and consent was obtained from all patients prior to the tests. SPAC was used for measuring the anterior chamber depth (ACD) in both eyes of the enrolled patients. In the case that both eyes satisfied the inclusion criteria, the left eye was employed for measurement.

\section{Subjects}

\section{Sensitivity and specificity evaluation group}

All glaucoma patients examined at the outpatient clinic of the Department of Ophthalmology, University of Yamanashi Hospital from February through May 2003 were enrolled. They underwent various examinations, including acquisition of information on the history of eye disease, determination of systemic complications, visual acuity test, IOP measurement, slit-lamp examination, gonioscopy, static automated visual field test or kinetic visual field test, optic disc examination and ultra biomicroscopic examination (UBM) by a glaucoma specialist prior to SPAC measurement, and were diagnosed for the type of glaucoma. The patients had undergone their most recent slit-lamp examination, gonioscopy or IOP measurement within 3 months prior to SPAC measurement, and undergone visual field tests within 6 months prior to SPAC measurement. The most recent eye examinations and SPAC measurement were performed without discontinuing all eye medications in current use. As eyes with aphakia or pseudophakia are known to have deeper ACD than eyes with phakia, we eliminated the eyes with aphakia or pseudophakia from the study.

\section{Classification of patients}

The patients were divided into the following three groups.

\section{ACG group}

Patients belonging to the ACG group were those who had an onset of ACG or those at a high risk of onset, who should be diagnosed with closed or narrow angle in regular health examinations. This group consisted of patients with primary ACG (PACG) eyes and their fellow eyes exhibiting a narrow angle, and eyes with PAC subjected to prophylactic LI. The standards for the indication of prophylactic LI consisted of a Shaffer's classification of grade 0 or 1 in a majority of the angle and provocative test positive eyes with an IOP elevation of $6 \mathrm{mmHg}$ or more in the prone position test, peripheral anterior synechia (PAS) or appositional angle closure, and at least one of the following: IOP elevation of more than $21 \mathrm{mmHg}$ or glaucomatous optic disc cupping with corresponding visual field defect, confirming the results of multiple reliable static visual field tests. However, patients with pseudophakia or aphakia, or those in whom SPAC measurement was difficult to perform, were excluded from this group, as shown in Table 1.

\section{Negative control group}

Patients whose eyes did not fit the aforementioned definitions of high-risk ACG eyes and were not at a risk of ACG onset were categorized as patients with control eyes. Patients satisfying the conditions listed in Table 1 were excluded from the study.

\section{Positive control group}

In all, 20 patients with ACG or PAC eyes who visited Kashiwagi Ophthalmic Clinic were enrolled in this study.

Table 1 Exclusion criteria

1. When fixation was judged to be improper because the alignment fixation lamp could not be observed at the center of the cornea

2. When focus was judged to be improper as a result of failure to superimpose the slit-lamp light and the reflected image of the fixation indicator

3. When consecutive images could not be photographed because the subject blinked or moved during photography

4. When slit-light observation was inadequate due to improper eyelid opening resulting in the photography of eyelashes and so forth

5. When it was difficult to identify the anterior surface of the iris with the slit-lamp microscope due to extreme mydriasis

6. When it was difficult to adequately observe the anterior chamber with the slit-lamp microscope due to low corneal transparency

7. When there were excessive irregularities on the cornea surface such as those following corneal transplant

8. Eyes with aphakia or pseudophakia 
Chronic ACG eyes prior to LI, fellow eyes of acute ACG eyes prior to LI, and eyes with PAC prior to LI in which appositional angle closure or PAS was observed at least in part of the angle, were classified as the positive control group separate from the sensitivity and specificity evaluation group, for the purpose of threshold analysis. The exclusion criteria for fellow eyes of PACG eyes employed for this group were as follows: eyes into which an intraocular lens (IOL) was inserted, cases of prominent paralytic mydriasis following an attack that led to difficulty in imaging, presence of intraocular diseases other than mild cataract, and cases of secondary ACG.

\section{SPAC measurement}

The system configuration, principle for measurement and data analysis were described in a previous paper. ${ }^{8}$ The system scanned the ACD from the optical axis to the limbus in approximately $0.66 \mathrm{~s}$ and took 21 consecutive slit-lamp images at $0.4 \mathrm{~mm}$ intervals. The system is completely noncontact and allows for the quantitative measurement of peripheral ACD. The SPAC measurement of ACD was performed three times and the mean of the ACD values at each point was taken to be the ACD for that point, as described in a previous report. ${ }^{8,9}$ Three physicians and two health care staffs who are familiar with the operation of SPAC measured the ACD in both eyes.

\section{Evaluation of sensitivity and specificity}

Sensitivity and specificity were examined according to the procedure described below using the measurement results. As was previously reported, ${ }^{8,9}$ because the most peripheral ACD that can be measured with this analyzer is normally approximately $6 \mathrm{~mm}$ from the pupil center, and ACD can usually be measured $1.2 \mathrm{~mm}$ from the pupil centre in this study, the ACDs were obtained at points ranging from 1.2 to $6.0 \mathrm{~mm}$ from the pupil centre.

\section{Discriminant analysis}

In order to confirm the advantage of diagnosis using multiple points, two kinds of discriminant analysis were performed with the SPAC data that were obtained by measuring ACDs at all points ranging from 1.2 to $5.6 \mathrm{~mm}$. One analysis used the data at all points, and the other used the data at 2.0 or $2.4 \mathrm{~mm}$.

\section{Threshold analysis}

The results of the foregoing analysis were found to be very useful only when all points were measured. However, there were some missing points in the clinical field due to focal corneal opacity, elongating or hanging cilia, or blinking. Thus, in order to formulate the criteria with consideration of the missing points, sensitivity analysis was performed with the threshold level of each point, which was determined from the mean and the standard deviation derived from the data of the positive control group. The equation for determining each threshold level was: 'mean $+z \times$ standard deviation,' where ' $z$ ' is the percentile of standard normal distribution. Two types of criteria were fomulated and sensitivity analyses were conducted by combining these criteria. One type of criteria was based on the number of 'bad' points that were below the threshold level at each point. For example, if the values at 2.0 and $2.4 \mathrm{~mm}$ were below their respective threshold levels, the patient would have two bad points. All eyes were divided into the four categories: category ' $x$ ' included eyes in which more than ' $x$ ' points were below their respective threshold levels. If categories 1 and 2 were defined as high-risk ACG and patients have eyes classified as category 1 or 2, these patient were classified as having eyes with a high risk of ACG. The other type of criteria was based on the value of ' $z$ '. Varying ' $z$ ' means varying the confidence interval (CI) at each point. Therefore, four receiver operated characteristics (ROC) curves were drawn while varying ' $z$ ', according to each threshold level of 1, 2, 3, or 4 .

The Statistical Analysis System (SAS Institute Inc. Cary, NC, USA) was used for statistical analysis. All enrolled eyes were subjected to SPAC measurement and the analysis was performed on the left eye unless the left eye satisfied the exclusion criteria.

\section{Results}

\section{Patients for assessment of sensitivity and specificity}

A total of 552 glaucoma patients (246 men and 306 women) were examined at the outpatient clinic of the Department of Ophthalmology, University of Yamanashi Hospital during the measurement period. Of all the measured eyes, seven eyes of seven patients were anophthalmic and 16 eyes of 16 patients exhibited phthisis bulbi; thus, SPAC measurement was performed on a total of 1081 eyes. Among these, there were 64 eyes for which SPAC measurement was difficult to perform. Of the 64 eyes, images of the anterior chamber that permitted analysis could not be obtained from 19 eyes, whereas SPAC measurement was difficult in the remaining 45 eyes due to improper fixation resulting from low visual acuity. A breakdown of these cases is shown in Table 2.

The negative control group consisted of 504 patients (238 men and 266 women), the breakdown of which is as follows: 206 patients with POAG, 191 with NTG, 60 with 
Table 2 Subjects excluded from the analysis

\begin{tabular}{lll}
\hline 1. & Low corneal transparency & Five subjects \\
2. & Bullous keratopathy & Eight subjects \\
3. & Exotropia & Two subjects \\
4. & Irregularities on the cornea surface & One subject \\
& following corneal transplant & \\
5. & Aniridia & Three subjects \\
6. Improper fixation due to low visual acuity & 45 subjects \\
7. & Eyes with aphakia or pseudophakia & 68 subjects \\
\hline
\end{tabular}

SOAG, 39 with $\mathrm{OH}$, and eight with congenital glaucoma. There were 80 high-risk ACG eyes in 48 patients (16 eyes in eight men and 64 eyes in 40 women). Among these, there were 19 eyes with acute ACG (and one patient exhibiting binocular attack), 17 fellow eyes of PACG eyes, 20 narrow angle eyes, 14 chronic ACG eyes, and 10 eyes with the plateau iris syndrome.

\section{Positive control group}

In all, 20 eyes satisfied the conditions set by this study but did not belong to the aforementioned sensitivity and specificity evaluation group. ACD was found to decrease linearly with increasing distance from the pupil centre.

\section{Discriminant analysis}

The 271 patients in whom ACD could be measured at all points ranging from 1.2 to $5.6 \mathrm{~mm}$ from the pupil centre were divided into the following: 40 with ACG eyes and 231 with negative control eyes. Although all conditions gave good ROC curves and AUC values, the AUCs obtained using the data from a single point 2 or $2.4 \mathrm{~mm}$ from the pupil centre were 0.97435 and 0.97157 , whereas the AUC obtained using the data from all SPAC measured points was 0.98831 (Figure 1).

\section{Threshold analysis}

Threshold analysis revealed that all four conditions (measurements at one point, two points, three points, and four points below the minimum value of $\mathrm{CI}$ ) the positive control group gave good ROC curves. One of the

Figure 1 Discriminant analysis using a single measured point or multiple measured points. ROC curves and AUC values from the result using a single measured point at $2 \mathrm{~mm}$ from the pupil centre (a) or at $2.4 \mathrm{~mm}$ from the pupil centre (b), or from the result using all measured points from 1.2 to $5.6 \mathrm{~mm}$ from the pupil center were depicted. $\mathrm{ROC}=$ receiver-operated characteristic, $\mathrm{AUC}=$ area under the curve, $n=271$.
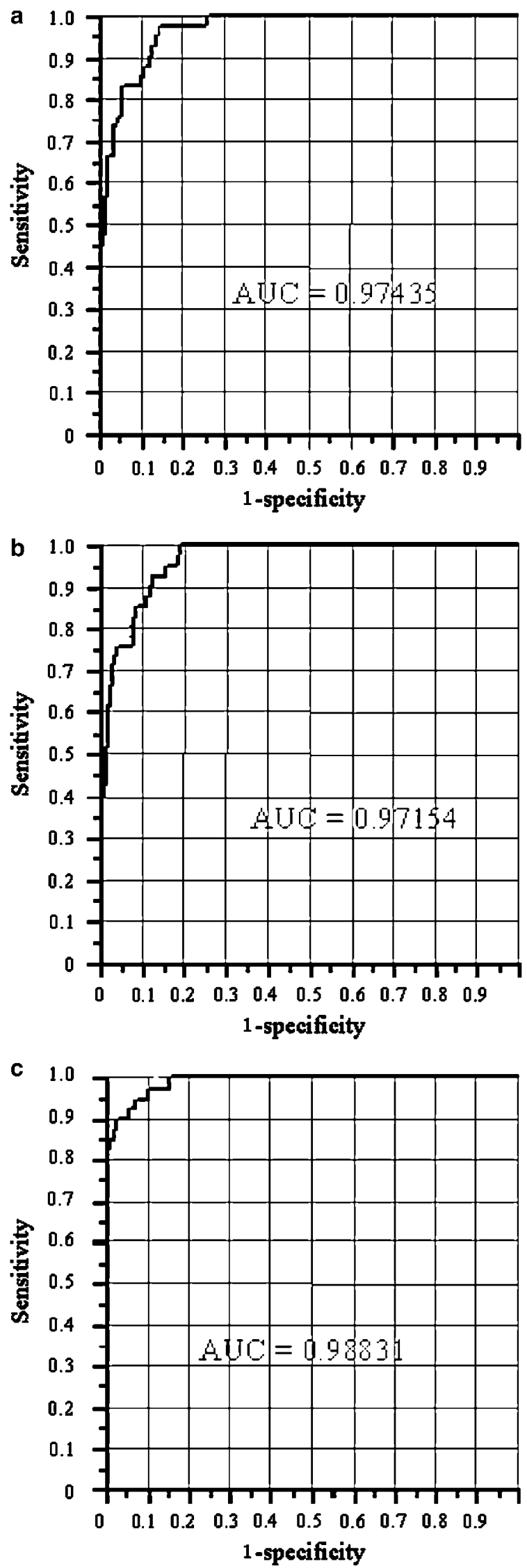


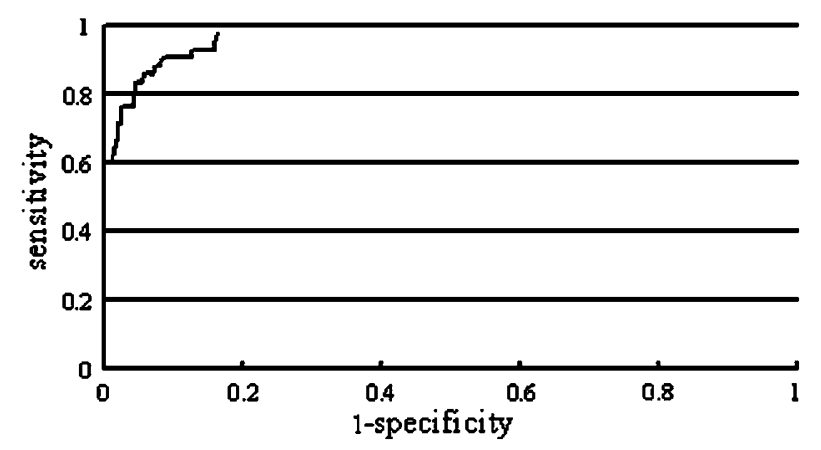

Figure 2 A representative ROC curve. This figure shows ROC curve when four measured points exceeded the threshold level. ROC $=$ receiver-operated characteristic, $n=552$.

good combinations for realizing high sensitivity and specificity under the four measurement conditions was the combination of the measurement of four points using CI of approximately 65-95\%, as shown in Figure 2. The sensitivities and specificities at $95 \% \mathrm{CI}$ at threshold levels $1,2,3$, and 4 were 1.00 and $0.61,0.98$ and $0.83,1.00$ and 0.70 , and 0.95 , and 0.98 , respectively. One of the most favourable conditions are a sensitivity of $97.6 \%$ and a specificity of $83.5 \%$, when data obtained from the four measured points exceeded their respective threshold levels determined at $95 \% \mathrm{CI}$.

\section{Discussion}

Although there are several studies of quantitative ACD measurement, ${ }^{10-15}$ their clinical usefulness is not consistent. The instruments or methods employed are expensive and difficult to handle, require complicated quantitative analysis, and have low reproducibility. Moreover, they are designed to be operated by ophthalmologists. Therefore, those methods are not suitable for screening eyes that are at a high risk of developing ACG and are not widely used today.

SPAC was developed for the purpose of screening eyes with narrow angle by health care staff other than ophthalmologists. ${ }^{8}$ As SPAC employs noncontact measurement, is noninvasive and simple to perform, and generates evaluations automatically, thereby allowing detecting ACG or PAC eyes by health care staff. We have indicated in our previous report that ACD can be measured with high reproducibility and the same measurement accuracy by both ophthalmologists and health care staff, ${ }^{16}$ that examinations can be performed in an extremely short period of $0.66 \mathrm{~s}$ per eye, and that the instrument itself is inexpensive due to its simple structure, thereby making it suitable for screening programs. ${ }^{8}$ Among the eyes assessed in this study, although there were 64 eyes (5.8\%) from which valid data could not be acquired, as low visual acuity and improper fixation can be assessed in regular health examinations, it is important to be able to detect eyes that have good visual function but are at a risk of ACG onset. In this study, the number of patients that could not be measured despite their having good visual acuity (better than 20/30) was extremely small (seven cases, 0.6\%). These findings also suggest that this instrument is suitable for use in screening procedures.

In order to eliminate bias among subjects, assessment was conducted on all patients who were diagnosed with glaucoma and were being examined at the outpatient clinic of the Department of Ophthalmology, University of Yamanashi Hospital for a certain period.

There are several reports of the sensitivity and specificity of ACG screening using ACD as an indicator. ${ }^{13,17-21}$ Devereux et al ${ }^{18}$ reported a sensitivity of $77 \%$ and a specificity of $83 \%$ in a study that used a slitlamp mounted ultrasound ACD measurement system. Congdon et al $^{17}$ reported a sensitivity of $88 \%$ and a specificity of $92 \%$ using ultrasonography in combination with tonography. Although no direct comparison can be made because different subjects are involved, the sensitivity and specificity obtained in this study are comparable to or better than those of the previous studies. One possible reason is that many of the conventional instruments yield measurement data from a single point, whereas the measurement data yielded by SPAC are obtained from multiple points. In the discriminant analysis using SPAC, the use of multiple measured points was clearly shown to yield favourable results.

In this study, we evaluated the ACD of glaucoma patients without having them discontinue their topical medications, including pilocarpine. The number of patients using pilocarpine eyedrops was very small (less than 3\%) and our results were very similar to that when the ACD data of patients who used pilocarpine eyedrops were eliminated from the analysis (data not shown).

This system has several limitations. As the system is an optical one, it is impossible to observe down to the bottom of the anterior chamber angle and to detect peripheral anterior synechia. In addition, only ACD in the outside direction can be evaluated, and measurement at peripheral regions is difficult for patients with prominent peripheral corneal opacity.

Taken together, the results of this study reveal that SPAC is useful for screening for eyes with narrow angle by health care staff other than ophthalmologists. Efforts should be made in the future to employ this instrument in regular health examinations, to confirm its usefulness, and to aid in the discovery of as many eyes at a risk of ACG as possible. 


\section{Acknowledgements}

We would like to express our sincerest appreciation to Messrs. Koji Tagawa and Junji Nakagawa for excellent technical assistance.

\section{References}

1 Erie JC, Hodge DO, Gray DT. The incidence of primary angle-closure glaucoma in Olmsted County, Minnesota. Arch Ophthalmol 1997; 115: 177-181.

2 Foster PJ, Baasanhu J, Alsbirk PH, Munkhbayar D, Uranchimeg D, Johnson GJ. Glaucoma in Mongolia. A population-based survey in Hovsgol province, northern Mongolia. Arch Ophthalmol 1996; 114: 1235-1241.

3 Foster PJ, Oen FT, Machin D, Ng TP, Devereux JG, Johnson GJ et al. The prevalence of glaucoma in Chinese residents of Singapore: a cross-sectional population survey of the Tanjong Pagar district. Arch Ophthalmol 2000; 118: 1105-1111.

4 Marchini G. Biometric data and pathogenesis of angle closure glaucoma. Acta Ophthalmol Scand Suppl 2002; 236: 13-14.

5 Quigley HA. Number of people with glaucoma worldwide. Br J Ophthalmol 1996; 80: 389-393.

6 Seah SK, Foster PJ, Chew PT, Jap A, Oen F, Fam HB et al. Incidence of acute primary angle-closure glaucoma in Singapore. An island-wide survey. Arch Ophthalmol 1997; 115: 1436-1440.

7 Foster PJ, Buhrmann R, Quigley HA, Johnson GJ. The definition and classification of glaucoma in prevalence surveys. Br J Ophthalmol 2002; 86: 238-242.

8 Kashiwagi K, Kashiwagi K, Toda Y, Osada K, Tsumura T, Tsukahara S. A newly developed peripheral anterior chamber depth analysis system - principle, accuracy, and reproducibility. Br J Ophthalmol 2004; 88: 1029-1034.

9 Kashiwagi K, Abe K, Tsukahara S. Quantitative evaluation of changes in anterior segment biometry by peripheral laser iridotomy using newly developed scanning peripheral anterior chamber depth analyzer. Br J Ophthalmol 2004; 88: 1035-1040.
10 Kondo T, Nakatsu A, Masami P. A method of image analysis for primary angle closure glaucoma. Ophthalmologica 1995; 209: 113-116.

11 Richards DW, Russell SR, Anderson DR. A method for improved biometry of the anterior chamber with a Scheimpflug technique. Invest Ophthalmol Vis Sci 1988; 29: 1826-1835.

12 Dragomirescu V, Hockwin O. Rotating slit image camera TOPCON SL 45. New developments for simultaneous image acquisition by photographic and CCD-assisted on-line documentation. Ophthalmic Res 1996; 28: 102-108.

13 Van Herick W, Shaffer RN, Schwartz A. Estimation of width of angle of anterior chamber. Incidence and significance of the narrow angle. Am J Ophthalmol 1969; 68: 626-629.

14 Jacobs IH. Anterior chamber depth measurement using the split-lamp microscope. Am J Ophthalmol 1979; 88: 236-238.

15 Lee DA, Brubaker RF, Ilstrup DM. Anterior chamber dimensions in patients with narrow angles and angleclosure glaucoma. Arch Ophthalmol 1984; 102: 46-50.

16 Osada K, Kashiwagi K, Tagawa K, Nakayama J, Tsukahara S. Reproducibility of a newly developed peripheral anterior chamber depth analysis system. Rinsho Ganka (Jpn J Clin Ophthalmol) 2004; 58: 715-718.

17 Congdon NG, Quigley HA, Hung PT, Wang TH, Ho TC. Screening techniques for angle-closure glaucoma in rural Taiwan. Acta Ophthalmol Scand 1996; 74: 113-119.

18 Devereux JG, Foster PJ, Baasanhu J, Uranchimeg D, Lee PS, Erdenbeleig Tet al. Anterior chamber depth measurement as a screening tool for primary angle-closure glaucoma in an East Asian population. Arch Ophthalmol 2000; 118: 257-263.

19 Vargas E, Drance SM. Anterior chamber depth in angleclosure glaucoma. Clinical methods of depth determination in people with and without the disease. Arch Ophthalmol 1973; 90: 438-439.

20 Zhang SF. Measurement of the depth of the anterior chamber in primary glaucoma and its clinical application. Zhonghua Yan Ke Za Zhi 1983; 19: 12-16.

21 Alsbirk PH. Limbal and axial chamber depth variations. A population study in Eskimos. Acta Ophthalmol (Copenh) 1986; 64: 593-600. 